\title{
Deep Brain Stimulation for DYT3 Dystonia
}

Alexander Lehn, MD, FRACP, ${ }^{1,2, *}$ Caroline Airey, FRACP, ${ }^{1}$ Sarah Olson, FRACS, ${ }^{3}$ John D. O'Sullivan, MD, FRACP, ${ }^{2,4}$ Richard Boyle, FRACP

Keywords: dystonia, parkinsonism, deep brain stimulation, globus pallidus internus.

DYT3 dystonia or "Lubag" is an X-linked recessive adult-onset movement disorder, first described in Filipino males from the Panay Islands in 1975. It manifests predominantly with dystonia in combination with parkinsonism.

Disease onset is usually in the late thirties with focal dystonia and progressive generalization in the majority of patients. In early stages, progressive axial dystonia is common. Parkinsonism can be a presenting feature, particularly in milder cases. After several years, the dystonic movements usually become less marked and stiffening of the limbs and straightening of the trunk occurs; this is the "combined dystonia/parkinsonian phase." After 15 years, parkinsonism usually predominates. Mean age at death is 55 years. ${ }^{1}$

Symptomatic treatment for this disease has been limited with partial response to standard therapy for dystonia, although zolpidem provided benefit in some patients. ${ }^{2}$ Dopaminergic therapy is usually unhelpful for the dystonia, but might help the parkinsonism. ${ }^{3}$ Recently, DBS of the globus pallidus internus (GPi) emerged as a potentially useful therapeutic option in small series. $^{4}$

We describe the case of a 53-year-old man from the Philippines who emigrated to Australia at age 42. He is the first genetically linked case of DYT3 dystonia reported in Australia. $^{5}$

The patient had normal birth and developmental milestones and was well during childhood and early adulthood.

Symptoms began at age 39 with subtle shuffling and slowing of his gait. By age 41, he had developed a resting tremor and dystonic posturing of his left upper limb as well as torticollis. Mobility became increasingly difficult over the subsequent years because of retrocollis and truncal hyperextension. Constant tongue protrusion was first noted at age 46, causing progressive dysarthria and dysphagia. In the years leading up to the patient's surgical intervention, he had developed chronic disabling back and neck pain and further deterioration in his gait with frequent falls, despite using a gait aid. At this stage, his speech was almost unintelligible and his dysphagia progressed to a degree where he required dietary modification.

MRI of the brain did not reveal any abnormalities.

At the time of surgery, in 2011, he was taking the following medication and daily doses, which had been added successively to provide modest symptomatic benefit: Pramipexole $3 \mathrm{mg}$ (some improvement of parkinsonian symptoms); lorazepam $6 \mathrm{mg}$ (modest benefit); lithium $675 \mathrm{mg}$ (good effect on axial rigidity); benzhexol $15 \mathrm{mg}$ (some effect, but limited by drowsiness); and baclofen $175 \mathrm{mg}$ (some effect, but limited by drowsiness).

Previous medications that had been trialed included pimozide, haloperidol, tetrabenazine, zolpidem, clonazepam, temazepam, diazepam, levodopa, and botulinum toxin.

Because of progressive symptoms, DBS electrodes (Medtronic, Minneapolis, MN) were implanted bilaterally targeting the GPi.

The patient's tongue protrusion resolved immediately after surgery preceding medication changes or activating the stimulator, and within 2 months postoperatively, his speech showed marked improvement, although he was still hypophonic. Also, the patient's generalized dystonia and upper-limb tremor had largely resolved. His gait had improved to allow him to mobilize unaided around his house. His chronic back pain had resolved. Unfortunately, though, the patient's dysphagia deteriorated further from baseline in the early postoperative period, necessitating the placement of a percutaneous endoscopic gastrostomy (PEG) tube.

At 12 months, his bulbar function had improved to a degree where his PEG tube could be removed and the patient was

${ }^{1}$ Department of Neurology, Princess Alexandra Hospital, Brisbane, Australia; ${ }^{2}$ Department of Neurology, Royal Brisbane and Women's Hospital, Brisbane, Australia; ${ }^{3}$ Department of Neurosurgery, Princess Alexandra Hospital, Brisbane, Australia; ${ }^{4}$ Royal Brisbane \& Women's Clinical School, University of Queensland, Brisbane, Australia

^Correspondence to: Dr. Alexander Lehn, Department of Neurology, Royal Brisbane and Women's Hospital, Brisbane, QLD, Australia; E-mail alex_lehn@health.qld.gov.au

Received 23 January 2014; revised 14 February 2014; accepted 19 February 2014.

Published online xx Xxxxx 2014 in Wiley InterScience (www.interscience.wiley.com). DOI:10.1002/mdc3.12017 
TABLE 1 Dystonia and parkinsonism scores at baseline and during follow-up

\begin{tabular}{|lllll|}
\hline Time point & BFMDRS score & BFMDRS relative change & UPDRS III score & UPDRS III relative change \\
\hline Pre-op off meds & 74 & - & 95 & $-70.5 \%$ \\
1 year on meds & 39.5 & $46.6 \%$ & 28 & $75.8 \%$ \\
2 years on meds & 12 & $83.8 \%$ & 23 & Resolution of tongue protrusion preceding activation \\
Latency of response to DBS & - Immediate improvement of dystonic posturing and upper-limb tremor \\
\multicolumn{2}{l}{ Stimulation parameters } & & Monopolar, 2.7 V, 250/240 $\mu \mathrm{s}, 150 \mathrm{~Hz}$ & \\
\hline
\end{tabular}

BFMDRS, Burke-Fahn-Marsden Dystonia Rating Scale.

eating a normal diet. He still had mild dysarthria, but no abnormal tongue protrusion.

Two years after surgery, the patient's symptoms continue to be well controlled. His dysarthria and hypophonia have improved significantly, and he now sings 1 hour every day. He mobilizes independently, although still has occasional freezing. Over the last 6 months, his parkinsonism has slightly deteriorated again, whereas the dystonia continues to improve. There is minimal dystonic posturing, no tremor, and only mild bradykinesia and rigidity. Over the second year, it has been possible to gradually withdraw lithium and pramipexole and reduce daily doses of lorazepam $3 \mathrm{mg}$, benzhexol $7.5 \mathrm{mg}$, and baclofen $75 \mathrm{mg}$.

Table 1 summarizes the dystonia and parkinsonism scores before surgery and during follow-up.

To date, there are 5 published cases of DBS for DYT3 dystonia, and all of them used GPi as the target. ${ }^{4}$ What is striking is the marked immediate improvement, particularly in dystonia, in all published cases.

In our case, as well as in 1 other case, improvement was noted even before switching the stimulation on, suggesting a microlesion effect. ${ }^{6}$ Typically, clinical benefit in primary dystonia is only observed weeks to months after activating the device, possibly related to preexisting high levels of neuroplasticity that form engrams of dystonic movements, which take time to normalize. ${ }^{7}$ The pathophysiology, and therefore the response to DBS in secondary dystonia, however, may differ, particularly when combined with other features, such as parkinsonism, as occurs in DYT3 dystonia.

The improvement in bulbar dysfunction was prominent and has been observed in other cases of primary and secondary dystonia, including Meige syndrome, tardive dystonia, and choreaacanthocytosis. Orolingual dystonia is often difficult to treat and causes significant morbidity, particularly in DYT 3 dystonia, so benefit from GPi DBS is especially noteworthy.

The benefit of DBS in secondary or degenerative dystonia is still subject to debate. The benefit in patients with DYT3 dystonia, demonstrated in this case to be maintained for at least 2 years, suggests that this therapy can provide a therapeutic option for such patients.

\section{Acknowledgments}

This publication did not require ethical approval. Written consent for publication was obtained from the patient involved.

\section{Author Roles}

(1) Research Project: A. Conception, B. Organization, C. Execution; (2) Statistical Analysis: A. Design, B. Execution, C. Review and Critique; (3) Manuscript: A. Writing of the First Draft, B. Review and Critique.
A.L.: 3A
C.A.: $3 B$
S.O.: $3 \mathrm{~B}$
J.O.S.: 3B
R.B.: $3 \mathrm{~B}$

\section{Financial Disclosures}

Nothing to declare.

\section{References}

1. Lee LV, Munoz EL, Tan KT, Reyes MT. Sex linked recessive dystonia parkinsonism of Panay, Philippines (XDP). Mol Pathol 2001;54:362-368.

2. Evidente VGH. Zolpidem improves dystonia in "Lubag" or X-linked dystonia-parkinsonism syndrome. Neurology 2002;58:662-663.

3. Lee LV, Kupke KG, Caballar-Gonzaga F, Hebron-Ortiz M, Müller U. The phenotype of the X-linked dystonia-parkinsonism syndrome. An assessment of 42 cases in the Philippines. Medicine (Baltimore) 1991;70:179-187.

4. Aguilar JA, Vesagas TS, Jamora RD, Teleg RA, Ledesma L, Rosales RL, et al. The promise of deep brain stimulation in X-linked dystonia parkinsonism. Int J Neurosci 2011;121(suppl 1):57-63.

5. Plummer C, Bradfield J, Singleton AB, Hernandez D, Singleton AA, O'sullivan J. First case report of X linked dystonia parkinsonism (XDP) or "Lubag" in Australia. J Clin Neurosci 2005;12:945-946.

6. Martinez-Torres I, Limousin P, Tisch S, Page R, Pinto A, Foltynie T, et al. Early and marked benefit with GPi DBS for Lubag syndrome presenting with rapidly progressive life-threatening dystonia. Mov Disord 2009:24:1710-1712.

7. Ruge D, Tisch S, Hariz MI, Zrinzo L, Bhatia KP, Quinn NP, et al. Deep brain stimulation effects in dystonia: time course of electrophysiological changes in early treatment. Mov Disord 2011;26:1913-1921.

\section{Supporting Information}

A video accompanying this article is available in the supporting information here.

Video. Segment 1 (2001): The major findings were neck and truncal dystonia. Note the jerky truncal hyperextension, particularly with walking. Segment 2 (2011, pre-op assessment): The patent had developed disabling constant tongue protrusion, in addition to increased parkinsonism with severe bilateral bradykinesia, rigidity, and rest and action tremor. His gait had 
become slow and shuffling with ataxia, persisting truncal dystonia, and requiring assistance with a gait aid. Segment 3 (2011, 2/12 post-op): Within 2 months of surgery, his tongue protrusion had resolved. Tremor and bradykinesia had improved significantly. His gait was more stable, although still required an aid. Segment 4 (2013): At 2 years, his parkinsonism remained improved, compared to the preoperative state. There had been no recurrent tremor or significant axial dystonia. His gait and turning was independent, although stride length remained reduced. 